Repair and Regeneration of the Nervous System, ed. J.G. Nicholls, pp. 187-202. Dahlem Konferenzen 1982. Berlin, Heidelberg, New York: Springer-Verlag.

\title{
Regeneration and Repair of the Nervous System: Clinical Aspects
}

\author{
H.-J. Freund* and H. J. Bauer** \\ *Neurologische Klinik der Universität Düsseldorf \\ 4000 Düsseldorf \\ **Neurologische Klinik der Universität Göttingen \\ 3400 Göttingen, F. R. Germany
}

\begin{abstract}
In man, restoration of function after damage to the central nervous system (CNS) cannot be achieved by regeneration. It is, however, frequently accomplished by survival of the damaged neurons. This is only possible if the lesion is of a reversible nature. If the disease process or injury has caused irreversible damage, functional recovery can be achieved by compensatory mechanisms mediated by structures that have escaped damage. Restitution of function due to redundancy in a distributed system and substitution of function by the remaining part of the system represent further mechanisms underlying functional recovery after irreversible damage.
\end{abstract}

At the clinical level there are no reliable criteria for distinguishing reversible from irreversible lesions at the acute stage. It is therefore difficult to assess the extent of restoration of function after stroke or other acute lesions. Positron emission tomography seems to have the potential for making such a distinction. This would provide the basis for taking measures to prevent the progression of a potentially reversible into an irreversible lesion. The astonishing ability of the brain to survive complete ischemia for a time of as long as one hour and partial ischemia for much longer is an example of a potentially reversible lesion in acute stroke. The capacity for late structural repair is illustrated in the restoration of conduction in demyelinated central axons. At present, therapeutic strategies along these lines promise more benefit than attempts to achieve regeneration of destroyed tissue. However, the inroads made by experimental investigations into regeneration in the CNS justify intensive clinicopathological studies of this problem. In contrast to the poor regenerative capacity of the CNS, successful structural and functional repair mechanisms are illustrated by the remarkable ability of the peripheral neurons to regenerate. 


\section{INTRODUCTION}

This background paper lists a sequence of problems related to restoration of function after damage to the nervous system as seen by the clinical neurologist. The approach may seem crude to the basic scientist involved in the precise analysis and experimental study of the mechanisms and issues under consideration. However, recent results of basic research on regeneration hold promise of transition to a more hopeful viewpoint and may be useful to bridge the gap between basic research on regeneration and the present situation in clinical medicine.

Following damage to the nervous system, the clinical neurologist is able to identify the loss of functional capacity. It is more difficult to assess whether the functional deficit is due to irreversible cell death, destruction of nervous tissue, or to transient cell dysfunction caused by circulatory or metabolic disturbances. Normal function may be resumed if the severity or duration of the disturbance has not led to irreversible destruction of nervous tissue.

According to present concepts, restoration of function following damage, especially to the central nervous system, results predominantly from a reorganization of the complex functional patterns of CNS activity and the utilization of pathways that have remained intact. The hope for an improvement in recovery and restitution of function following damage to the nervous system rests on the development of a) more effective measures for preventing the progression from reversible towards irreversible lesions and b) possible means of achieving true regeneration. At the clinical level recovery of function by regeneration appears limited at the present time, and inroads can only be achieved by basic research. In the following presentation, some of the problems are considered in the light of clinical experience with patients in whom there is functional loss, probable damage to structure, and a possibility for restoration. In the peripheral nervous system, recovery by regeneration is a commonplace event, but even in these favorable circumstances restitution of function is often less than complete. 


\section{AXONAL REPAIR MECHANICS}

Although the interference of disease with structural integrity and function shows a broad spectrum regarding the site, extent, and nature of the lesion, the interest in functional recovery after damage to the nervous system has been focused on the regeneration and remyelination of axons. Much of this interest has been aroused by the fundamental difference between the capability for regeneration and remyelination in the central and peripheral nervous system. With respect to regeneration, it has been suggested that a key difference may lie in the sheath structures through which the regenerating axons must pass. These may be stronger in peripheral nerves because of the presence of the basal lamina and may, therefore, resist the expanding pressure of the terminal club, forcing it to grow towards the discontinuity according to this hypothesis. In the central nervous system the myelin sheath is unable to restrain the expanding force of the terminal club, resulting in continuous expansion and rupture $(3,31)$. Remyelination is more successfully achieved by Schwann cells in peripheral nerve fibers than by the oligodendrocytes in the CNS. A differential role of the growth-associated proteins also seems possible (see Willard, this volume).

\section{REGENERATION OF AXONS}

A classic example for the study of the regeneration of axons in the CNS is spinal cord transection (see Mendell, this volume). In addition to spinal trauma, cord transection may occur following hemorrhages due to vascular malformations, in severe necrotic myelopathy, and in space-occupying lesions. One factor responsible for the prevention of regrowth of axons is the proliferation of glial cells. Glial cells form a dense tangle in the damaged area of the spinal cord and obstruct the regeneration of axons. Various procedures have been tried in order to overcome this blockade of regenerating axons $(1-4,20)$. Since Schwann cells serve to direct axon regrowth, Aguayo (this volume) attempted to overcome the blockade to spinal axon regeneration by removing the damaged portion of the cord and replacing it with a graft of peripheral nerve tissue. Some spinal axons 
crossed the junction between the cut end of the spinal cord and grew distances of up to $10 \mathrm{~mm}$. It remained unclear, however, whether the spinal axons reentered the cord on the other side of the graft and made connections. Functional improvement was not seen in any case. Guth and co-workers (12) have recently summarized the requirements for proving restoration of function by regeneration: a) processes of central nervous system neurons must bridge the level of injury, b) the regenerated fibers must make synapses, c) the regenerated fibers must generate post-junctional responses, and d) changes in function must derive from regenerated connections. Only when these criteria are met is there proof that a return of function is due to the reconnection of severed parts of the spinal cord.

It is clear that these postulates cannot be applied to clinical studies on the mechanisms underlying the restoration of function after damage to the human central nervous system. The usual clinical approach in looking at recovery of function is, therefore, to attribute every functional improvement to mechanisms other than true regeneration as long as Guth's criteria are not fulfilled by animal experiments on the adult mammalian central nervous system. For complete spinal cord transection in man, there is no evidence for functional recovery, by whatever mechanism.

An example of successful sprouting in the mammalian CNS has been shown by experiments on central cholinergic denervation in the rat (8). Following injury to an identified cholinergic pathway, sympathetic axons arising from the superior cervical ganglion, which are normally confined to extracerebral structures and blood vessels, invaded those brain areas denervated of cholinergic fibers. Afferent input to the sprouting fibers is not required, so that the signal for regrowth must come from the denervated tissue. The functional implication of such an innervation is unclear. Nevertheless, this model is interesting because of the specificity of the neuronal management with directed growth in response to a specific signal. 
Since little is known about an increasing number of disorders of the human central autonomic system, further study of such mechanisms would be interesting.

REMYELINATION AND RESTORATION OF CONDUCTION IN CENTRAL AXONS Another major issue for the study of restoration of function concerns demyelination and remyelination of axons in the central nervous system. The model disease is multiple sclerosis, but there are various other demyelinating agents: lysolecithin, diphtheria toxin, CSF-barbitage, cold injury, pressure or virusinduced demyelination produced by mouse corona virus JHM, canine distemper virus, Theiler virus, visna, or experimental allergic encephalomyelitis produced by brain/spinal cord tissue or their components with Freund's adjuvant. These lesions allow the study of remyelination in the CNS under experimental conditions. Such studies have provided insight into the mechanisms of conduction blockade after demyelination. It has been shown $(6,7,26)$ that conduction along demyelinated zones can be restored. The appearance of continuous conduction along such segments indicated the appearance of sodium channels in the internodal membrane. Their formation requires about six days and has been attributed to either newly synthetized protein or lateral movement of nodal elements. The restoration of conduction in demyelinated segments is usually associated with limited high frequency response capability.

Attempts to improve the reliability of transmission across a demyelinated zone by prolongation of the action potential have been pursued. Pharmacologically, 4-aminopyridine, and tetraethylammonium increase conduction in demyelinated segments (5, 7). Clinical trials were complicated by the occurrence of seizures which prevented their further administration. Polypeptide neurotoxins (e.g., scorpion toxin) may also improve conduction across demyelinated zones, but their clinical use is doubtful because of difficulties in crossing the blood-brain barrier and because of side effects. 
The failure of axons to acquire new myelin in MS may be due to the destruction of oligodendrocytes formerly providing myelin in the damaged area. Competent oligodendrocytes may be unable to divide in the adult CNS and to migrate into the lesion. The recovery of function in MS, as far as can be attributed to repair mechanisms of the damaged fibers, is therefore largely due to the recession of edema and/or the restoration of conduction in demyelinated fibers rather than to remyelination. Remyelination has been shown to play an important role in restoring conduction in cat spinal cord axons demyelinated by microinjection of lysophatidyl choline (27). In the human CNS, restoration of conduction by remyelination is likely to contribute to recovery from compressive and traumatic lesions of the central nervous system but plays little part in the remission of multiple sclerosis (27). The correlation between the results of experimental demyelination and the clinical features of MS have considerably promoted our understanding of this possibly immunological disease, which is characterized by acute episodes with manifestations of the deficits and their remission within a period of a few days to several weeks. Demyelinating diseases illuminate the role of neural and glial cell interaction and were discussed in detail at one of the Dahlem Workshops on neuronal-glial interactions in 1980 (25).

The evidence obtained from investigations of regeneration and remyelination of damaged axons in the CNS indicates that several factors may prove promising by future attempts to improve latent capabilities. But at this time they do not contribute significantly to the functional recovery in the human cNS.

\section{PERIPHERAL NERVOUS SYSTEM INJURY}

In the peripheral nervous system it is customary to grade the effects of acute nerve injury as follows: a) rapidly reversible "physiological" or metabolic block not associated with anatomical changes, b) segmental or paranodal demyelination, c) Wallerian degeneration, and d) irreversible necrosis (9). Such acute injuries to nerve fibers may be due to physical forces (e.g., percussion, compression, angulation, or stretch) 
or to sudden environmental changes (e.g., ischemia, acute metabolic disturbance, or cold injury).

The prognosis depends upon the severity and duration of the insult. In a mild nerve compression syndrome, for example, a demyelinating block may recover completely within a few weeks, whereas severe compression (such as that sustained during prolonged drug coma) may result in irreversible necrosis of both muscle and nerve at the site of pressure.

In chronic or recurrent nerve injury, as in entrapment syndromes, the situation is complicated by the hypertrophic Schwann cells and off-reaction of the connective tissue components of the nerve trunk, giving rise to neuroma formation (9). In this situation there may also be secondary effects on the distal axon (32), which render it more susceptible to further damage (the "double crush" syndrome (30)). Inherited or metabolic disorders can also make peripheral nerves more susceptible to physical injury. Technical factors, such as the level of injury, duration of denervation, or the necessity for graft insertion, are of obvious prognostic importance and are well recognized in specific clinical situations (28).

FUNCTIONAL RECOVERY AFTER REVERSIBLE LESION OF CENTRAL NEURONS The poor ability of the mammalian CNS to restore disrupted connections between neurons raises questions concerning the mechanism for functional recovery. For the clinician, the most important and challenging mechanism underlying functional recovery after brain lesions is the restoration of cell function. This is only possible if the damaged neurons survive and the dysfunction is reversible. In acute lesions it is particularly difficult to decide to what extent the functional impairment of the patient is reversible or irreversible in nature. The problem of functional recovery in the human CNS will therefore be discussed separately as a) the earlier repair mechanisms after reversible cell damage, and b) the remaining mechanisms after irreversible cell damage. Compensatory changes, 
redundancy in distributed systems, relearning, and sparing of function in early lesions are candidates for the restoration of function not dependent on structural repair.

Since destroyed neurons cannot regenerate, the only therapeutic possibility rests with measures preventing irreversible destruction of cells during the critical time. The investigation of the mechanisms underlying the recovery of cells after potentially reversible lesions represents, at the present time, the most promising potential for therapeutic progress. The issue is how to prevent the progression of the pathological process from a reversible to an irreversible lesion. At the acute stage of an injury to the brain or of a disease such as encephalitis or stroke, the extent of functional impairment may be dramatic. After periods of unconsciousness, severe neurological, and psychopathological deficits, full recovery may follow in some cases. The time course of such improvement may show rapid early recovery, but it is sometimes delayed for months. It is possible, at least in some patients, to ensure that such recovery is mainly the consequence of restoration of activity in the damaged cell systems.

The astonishing ability of nerve cells to survive major interference with their normal environment has been shown in studies on cerebral ischemia. Ischemic brain infarcts represent the most frequent neurological disease. In the general population, the stroke mortality ranges only a little behind that of heart infarction. The occlusion of one or more major brain arteries may lead to ischemic brain infarctions. Although the territories supplied by the cerebral arteries are well-defined, the sizes of the infarcts vary considerably between massive infarctions causing major permanent neurological deficits and minimal infarcts with little or no deficit. Several factors are responsible for this variability: collateral capacities by anastomoses, the hemodynamic and nutritional situation, and preset factors at the time when the stroke occurs. The factors that could be manipulated during the early phase of acute therapy are related to the development of brain swelling by ischemic 
edema. Ischemic brain swelling is the major determinant for morbidity and mortality from stroke. It has been shown by experiments in cats and monkeys that the cerebrum can survive complete ischemia of the brain lasting for one hour (15). The restoration of aerobic glycolysis is indeed surprising after such prolonged ischemia. This progresses in three stages towards recovery: suppressed oxygen and glucose consumption rates during early resuscitation lasting up to one hour, a subsequent period of increased glycolysis lasting a few hours, and then the attainment of pre-ischemic values. The functional outcome depends on the secondary complications associated with such ischemic shock: the no-reflow phenomenon, post-ischemic hypertension, post-ischemic brain swelling, disturbance in cerebrovascular reactivity, coagulopathy, and pulmonary congestion. Where these variables take a favorable course, the functional outcome may be surprisingly good and long-lasting survival is possible.

The factors determining the development of post-ischemic edema can also be examined under the conditions of graded reduction of cerebral blood flow. This condition mimics the clinical situation more realistically than complete cessation of brain circulation. When regional blood flow was reduced below a critical value of $10-15 \mathrm{ml} / 100 \mathrm{~g} / \mathrm{min}$, brain edema and stroke developed which caused microcirculatory compression and further decrease in blood flow (24). If the blood flow was kept above this threshold for critical ischemia, neither brain edema nor functional impairment developed. Therapeutic measures for the prevention of the development of stroke therefore aim to influence flow values before they reach critical levels. Clinically, the first two pathogenetic mechanisms are treated by the infusion of hyperosmolaric substances and high-dose corticosteroids. Barbiturates may promise a protective function in lowering the threshold of critical ischemia.

The various mechanisms of brain metabolism and function associated with changes in cerebral blood flow have been investigated in great detail. It is therefore safe to conclude that 
the brain is able to survive and to restore metabolic and physiological function after much longer periods of critical ischemia than the human brain is able to survive after cardiac arrest. This difference between cats and monkeys on the one hand and human patients on the other is largely unexplained, but it may be at least partly due to the differences attributable to cessation of the total circulation in man as compared with intact body circulation in the animal experiments. In the case of regional ischemia, the minimal stroke model holds considerable promise for the treatment and prevention of stroke.

The difficulty of the clinician in the acute stage of a disease or at the time when the patient's symptoms culminate is to judge to what extent the lesion is reversible or irreversible. A patient with a hemiparesis may have the full spectrum of functional outcome ranging from complete, persistent hemiparalysis to full functional recovery. This difficulty remains true for the subsequent period since the recovery of function provides no clues about the relative contributions of restoration of cell function and compensatory mechanisms. Recovery of function cannot be used as an indicator in making such a distinction. It may be due to the restoration of function in a population of neurons which were damaged transitorily. This would be the case in a reversible lesion. In an irreversible lesion, recovery of function must be due to compensatory changes by neuronal populations outside the persistently damaged area.

Until recently there has been no technique that enabled a distinction to be made between a reversible and an irreversible lesion or to reveal its extent. Neither the angiogram nor the CT scan is helpful in this respect. Tumors, infarcts that are older than a few days, and intracerebral bleeding can be revealed by СT. The damaged areas in inflammatory and metabolic disorders usually cannot be seen, except for late sequelae in some cases. The first new technique that seems to provide the potential to distinguish reversible from irreversible lesions is Positron-emission-tomography (PET). This method is described 
by Crill and Raichle (this volume) and seems to be able to overcome the difficulties involved in the prediction of the restoration of neuronal function, especially in the case of stroke. It offers the possibility of relating brain function to blood flow and metabolism by measuring regional cerebral blood flow three-dimensionally and of monitoring glucose uptake, transport, and consumption in the brain area of interest. The resolution is limited, but it is good enough for most clinical problems.

The assessment of the reversible nature of a lesion by the PET technology opens new avenues for the development of rational therapies. At the moment there is a worldwide cooperative study exploring the therapeutic value of extra-intracranial bypass operations for the treatment and prevention of cerebrovascular diseases. The rationale for the indication is that the patient's symptoms should have been transitory and a major blood vessel obstruction responsible for these transient ischemic attacks has been shown by angiography. This approach is again hampered by the lack of knowledge about the structural integrity in the area of interest. PET-scanning will be able to provide information about whether an ischemic brain area with reversible cell damage can be rescued, or whether more blood will be supplied to a scar surrounded by a fringe of luxury perfusion. Partial cell dysfunction in generalized rather than in focal lesions due to inflammation or metabolic disorders will be more difficult to assess by such a technique.

The restoration of function on the basis of structural recovery of reversibly damaged nerve cells represents by far the most important acute repair mechanism of the central nervous system. Its diagnostic assessment and therapeutic manipulation hold considerable promise for prognosis and treatment. It provides a basis for the search for measures to prevent irreversible lesions.

\section{FUNCTIONAL RECOVERY AFTER IRREVERSIBLE CNS LESIONS}

This type of recovery has to be accomplished by the various mechanisms of compensatory changes, relearning, and unmasking 
of latent capacilities due to the activities of cells outside the damaged area. It is extensively discussed by Singer (this volume) and will therefore be dealt with only cursorily here. According to the Kennard $(17,18)$ principle, lesions acquired early in life are better compensated for than those occurring later. Whereas the importance of the critical period in mammals has been demonstrated in animals (16), in man the situation seems to be less clear. Teuber and Rudell (29) suggested that "Whether an early lesion produces less or more disruption effects than a later one, or effects that are equally disruptive, depends on the site and size of the lesion and on the nature of the tasks employed in establishing its effects." From subsequent work $(10,11)$, evidence was provided that the escape of function after early lesions depends on the maturation of the impaired system. The relative sparing of language after early lesions of the left hemisphere of young children as a consequence of a shift of language function from the left to the right hemisphere has often been referred to as an example of neural plasticity. It has, however, been shown that this shift is achieved at the price of a reduction in the capacities of the right hemisphere. This has been interpreted as a de-repression of a preexisting potential for language of the right hemisphere. It is difficult to decide from experiments producing defined lesions in animals whether the recovery of function as demonstrated behaviorally is due to structural and physiological rearrangements in the damaged area or to compensatory mechanisms including residual functional mechanisms. A further difficulty lies in the proper choice of the neural system to be explored by appropriate tests. Experiments on the restoration of function after carefully anatomically controlled lesions of different descendent motor pathways in the freely behaving monkey have provided little evidence for true functional recovery by whatever mechanisms $(22,23)$. Recovery of function does not occur in motor systems that do not share functions with other systems: after interrupting various systems sharing a function, little recovery ensues. Kuypers (21) concluded that apparent recovery may be the result of nonspecific testing that fails to show the loss of a specific function 
because the animal is allowed to employ different tactics in performing the task.

Comparing functional recovery in young and adult animals, Kling and Tucker (19) suggested the involvement of two types of shift mechanisms mediating the observed functional improvement: a) neurological shift in the adult achieved by an intact structure assuming the function of an ablated area, and b) behavioral shift in young animals accomplished by employing new strategies in the performance of the new task. In patients in whom the extent of the lesion is less-defined than in animals, the distinction between the various recovery mechanisms is in most cases unclear. Many disorders affect the CNS by widely distributed structural impairment. Assessment of recovery mechanisms is only possible in focal lesions. Gunshot wounds were extensively studied after the two world wars. It has been shown that interruption of brain pathways or damage of cortical areas show no functional recovery when an elementary function is disturbed. The clearest results come from follow-up studies on visual field defects following gunshot wounds. The scotomata remained absolutely unchanged for decades (14). The more widely spread the projection or cortical representation of a function (comparison of weights) and the more complex the cerebral dysfunction (aphasia, global cerebral dysfunction after head trauma), the more difficult the assessment of possible repair mechanisms involved. The examination of patients following division of the Corpus callosum for the treatment of intractable epilepsy is an example of the study of brain dysfunction and its recovery after interference with complex brain circuits. Whereas the structural repair mechanisms of peripheral nerves can achieve functional recovery on the basis of structural repair, for the human CNS the statement of Holmes (13) is still valid: "The truth of the Wallerian law, that portions of fibres separated from their trophic centres degenerate, is incontestible."

Acknowledgement. We are indebted to R.W. Gilliatt for his advice and help with the section on "Peripheral Nervous system Injury." 


\section{REFERENCES}

(1) Aguayo, A.J.; Bray, G.M.; Perkins, C.S.; and Ducan, I.D. 1979. Axon-sheath cell interactions in peripheral and central nervous system transplants. 1979 Soc. Neurosci. Symp. $\underline{4}: 361-383$.

(2) Aguayo, A.J.; Bunge, R.P.; Duncan, I.D.; Wood, P.M.; and Bray, G.M. 1979. Rat Schwann cells cultured in vitro can ensheath axons regenerating in mouse nerves. Neurology (Minneapolis) 29: 589 .

(3) Aguayo, A.J.; Dickson, R.; Trecarten, J.; Atwell, M.; Bray, G.; and Richardson, P. 1978. Ensheathment and myelination of regenerating PNS fibers by transplanted optic nerve glia. Neurosci. Lett. 9: 97-104.

(4) Aguayo, A.J.; Kasarjian, J.; Skamrnr, E.; Kongshavn, P.; and Bray, G.M. 1977. Myelination of mouse axons by Schwann cells transplanted from normal and abnormal human nerves. Nature (London) 268: 753-755.

(5) Bostock, H., and McDonald, W.I. 1982. Recovery of function after demyelination. In Proceedings of the Dahlem Workshop on Neuronal-Glial Interrelationships: Ontogeny, Maintenance, Injury, Repair. Berlin: Springer.

(6) Bostock, H., and Sears, T.A. 1978. The internodal axon membrane: electrical excitability and continuous conduction in segmental demyelination. J. Physiol. 280: 273-301.

(7) Bostock, H.; Sherrat, R.M.; and Sears, T.A. 1978. Overcoming conduction failure in demyelinated nerve fibres by prolonging action potentials. Nature 274: 385-387.

(8) Crutcher, K.A., and Davis, J.N. 1981. Sympathetic noradrenergic sprouting in response to central cholinergic denervation. Trends Neurol. Sci. 4/3: 70-72.

(9) Gilliatt, R.W. 1981. Physical injury to peripheral nerves. Physiologic and electrodiagnostic aspects. Mayo clin. Proc. 56: 361-370.

(10) Goldman, P.S. 1971. Functional development of the prefrontal cortex in early life and the problem of neuronal plasticity. Exp. Neurol. 32: 366-387.

(11) Goldman, P.S. 1972. Developmental determinants of cortical plasticity. Acta Neurobiol. Exp. 32: 495-511.

(12) Guth, L.; Brewer, C.R.; Collins, Jr., W.F.; Goldberger, M.E.; and Perl, E.R. 1980. Editorial commentary. Criteria for evaluating spinal cord regeneration experiments. Exp. Neurol. 69: 1-3. 
(13) Holmes, G. 1906. On the relation between loss of function and structural change in focal lesions of the central nervous system, with special reference to secondary degeneration. Brain 29: 514-523.

(14) Holmes, G., and Lister, W.T. 1916. Disturbances of vision from cerebral lesions, with special reference to the cortical representation of the macula. Brain 39: 34 .

(15) Hossmann, K.A., and Kleihues, P. 1973. Reversibility of ischemic brain damage. Arch. Neurol. 29: 375-384.

(16) Hubel, D.H., and Wiesel, T.N. 1970. The period of susceptibility to the physiological effects of unilateral eye closure in kittens. J. Physiol. 206: 419-436.

(17) Kennard, M.A. 1936. Age and other factors in motor recovery from precentral lesions in monkeys. Am. J. Physiol. 115: $138-146$.

(18) Kennard, M.A. 1940. Relation of age to motor impairment in man and in subhuman primates. Arch. Neurol. Psychiatr. 44: $377-397$.

(19) Kling, A., and Tucker, T.J. 1968. Sparing of function following localized brain lesions in neonatal monkeys. In The Neuropsychology of Development, ed. R.L. Isaacson, pp. 121-146. New York: John Wiley and Sons, Inc.

(20) Krikorian, J.G.; Guth, L.; and Donati, E.J. 1981. Origin of the connective tissue scar in the transected rat spinal cord. Exp. Neurol. 72: 698-707.

(21) Kuypers, H.G.J.M. 1974. Recovery of motor function in rhesus monkeys. Neurosci. Res. Prog. Bull. 12/2: 240-244.

(22) Lawrence, D.G., and Kuypers, H.G.J.M. 1968a. The functional organization of the motor system in the monkey. I. The effects of bilateral pyramidal lesions. Brain 91: $1-14$.

(23) Lawrence, D.G., and Kuypers, H.G.J.M. 1968b. The functional organization of the motor system in the monkey. II. The effects of lesions of the descending brain-stem pathways. Brain 91: 15-36.

(24) Schuier, F.J., and Hossmann, K.-A. 1980. Experimental brain infarcts in cats. II. Ischemic brain edema. Stroke 11: 593-601.

(25) Sears, T.A., ed. 1982. Neuronal-glial Cell Interrelationships. Dahlem Konferenzen. Berlin: Springer.

(26) Sears, T.A.; Bostock, H.; and Sherrat, R.M. 1978. The pathophysiology of demyelination and its implication for the symptomatic treatment of multiple sclerosis. Neurology 28: 21-26. 
(27) Smith, K.J.; Blakemore, W.F.; and McDonald, W.I. 1979. Central remyelination restores secure conduction. Nature 280: 295-396.

(28) Sunderland, S. 1978. Nerve and Nerve Injuries, 2nd ed. Edinburgh: Churchill, Livingstone.

(29) Teuber, H.-L., and Rudel, R.G. 1962. Behavior after cerebral lesions in children and adults. Dev. Med. Child. Neurol. $\underline{4}$ : 3-20.

(30) Upton, A.R.M., and McComas, A.J. 1973. Double crush in nerve entrapment syndromes. Lancet II: 359-361.

(31) Veraa, R.P., and Grafstein, B. 1981. Cellular mechanisms for recovery from nervous system injury: a conference report. Exp. Neurol. 71: 6-75.

(32) Weiss, P., and Hiscoe, H.B. 1948. Experiments on the mechanism of nerve growth. J. Exp. Zool. 107: 315. 